\title{
Towards a Robust, Universal Predictor of Gas Hydrate Equilibria by means of a Deep Learning Regression Supporting Information
}

\author{
Michael K.B. Landgrebe ${ }^{\mathrm{a}, *}$, Diakanua Nkazi ${ }^{\mathrm{a}}$ \\ ${ }^{a}$ School of Chemical and Metallurgical Engineering, Faculty of Engineering and the Built Environment, \\ University of the Witwatersrand, Johannesburg, South Africa. \\ *Corresponding Author. E-mail address: landgrebemkb@wkh.co.za
}


Model G Topology

3 Hidden Layer Neural Network

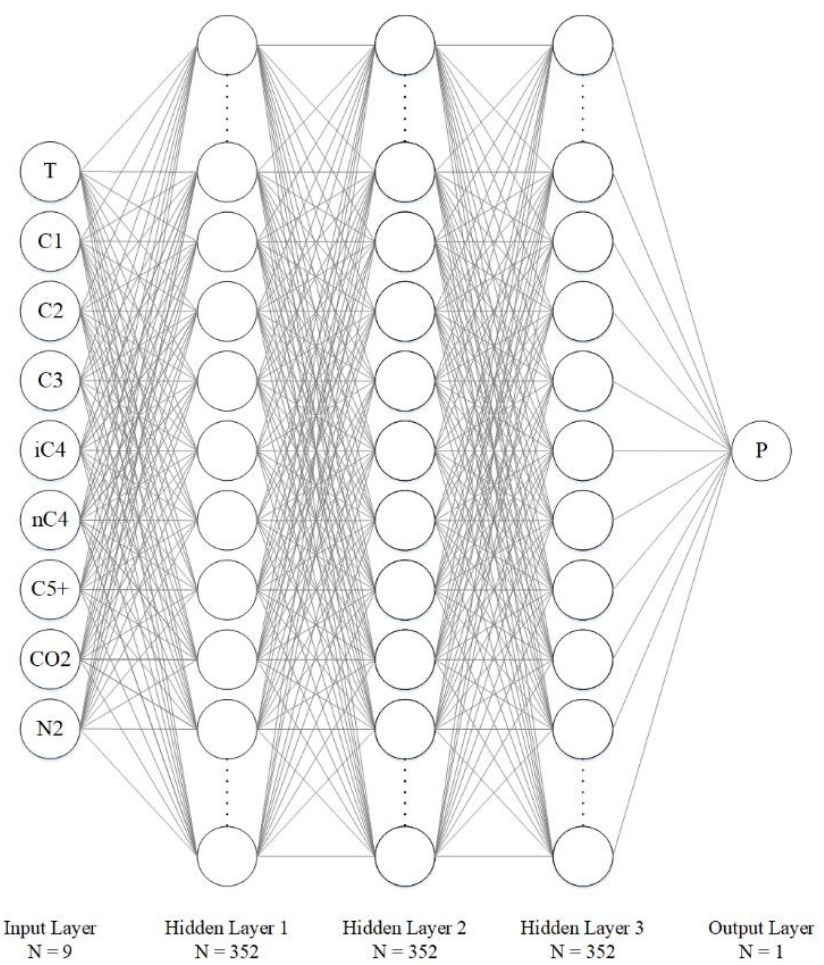

$\mathrm{N}=9 \quad \mathrm{~N}=352 \quad \mathrm{~N}=352 \quad \mathrm{~N}=1$

\begin{tabular}{|l|l|}
\hline \multicolumn{2}{|c|}{$\begin{array}{c}\text { Table S.1: Model G } \\
\text { Hyperparameters }\end{array}$} \\
\hline Paramter & Value \\
\hline Architecture & $\begin{array}{l}\text { Multi-Layer } \\
\text { Perceptron }\end{array}$ \\
\hline Hidden Layers & 3 \\
\hline $\begin{array}{l}\text { Neurons per } \\
\text { Hidden Layer }\end{array}$ & 352 \\
\hline $\begin{array}{l}\text { Hidden Layer } \\
\text { Activation }\end{array}$ & ReLU \\
\hline Loss & Mse \\
\hline Optimizer & Adam \\
\hline Epochs & 8000 \\
\hline Batch size & 256 \\
\hline $\begin{array}{l}\text { Cross-validation } \\
\text { Folds }\end{array}$ & 10 \\
\hline $\begin{array}{l}\text { Test split } \\
\text { (cross- } \\
\text { validation) }\end{array}$ & $30 \%$ \\
\hline $\begin{array}{l}\text { Validation split } \\
\text { (hold-out) }\end{array}$ & $10 \%$ \\
\hline $\begin{array}{l}\text { Hidden Layer } \\
\text { Drop-out }\end{array}$ & $5 \%$ \\
\hline
\end{tabular}

Figure S.1: Neural Network Layout

\begin{tabular}{|c|c|c|c|c|}
\hline \multicolumn{5}{|c|}{ Table S.2: Model G Expanded Layout } \\
\hline Layer (type) & Neurons & Activation & Drop-out (\%) & Initializer \\
\hline Input & 9 & - & 0 & - \\
\hline Hidden 1 & 352 & ReLU & - & Normal \\
\hline Dropout 1 & - & - & 5 & - \\
\hline Hidden 2 & 352 & ReLU & - & Norma I \\
\hline Dropout 2 & - & - & 5 & Norma I \\
\hline Hidden 3 & 352 & ReLU & - & - \\
\hline Dropout 3 & - & - & 5 & Normal \\
\hline Output & 1 & Linear & 0 & \\
\hline
\end{tabular}

\begin{tabular}{|c|c|c|}
\hline \multicolumn{2}{|c|}{ Table S.3: Model G Architecture } \\
\hline Layer (type) & Output Shape & Parameters \\
\hline Dense_41 & (None, 352) & 3520 \\
\hline Dropout_41 & (None, 352) & 0 \\
\hline Dense_42 & (None, 352) & 124256 \\
\hline Dropout_42 & (None, 352) & 0 \\
\hline Dense_43 & (None, 352) & 124256 \\
\hline Dropout_43 & (None, 352) & 0 \\
\hline Dense_44 & (None, 1) & 353 \\
\hline
\end{tabular}


The contents of this file are intended to serve as supplementary information for the paper titled "Towards a Robust, Universal Predictor of Gas Hydrate Equilibria by means of a Deep Learning Regression". Information regarding the neural network topology and list of parameters for training and testing the model is provided so as to permit replication of the methodology of this research in other works, be they in the field of gas hydrates or general machine learning regression application. The model has been developed using Python, using the machine learning library Keras running on a Tensorflow backend. Details on the exact methodology used, in addition to all data source references may be found in the paper itself. 